\title{
Recommendation on screening adults for asymptomatic thyroid dysfunction in primary care
}

\author{
Richard Birtwhistle MD MSc, Kate Morissette MSc, James A. Dickinson MBBS, Donna L. Reynolds MD MSc, \\ Marc T. Avey PhD, Francesca Reyes Domingo MHSc, Rachel Rodin MD, Brett D. Thombs PhD; for the Canadian \\ Task Force on Preventive Health Care
}

Cite as: CMAJ 2019 November 18;191:E1274-80. doi: 10.1503/cmaj.190395

This guideline is available in French at www.cmaj.ca/lookup/suppl/doi:10.1503/cmaj.190395/-/DC1

CMAJ Podcasts: interview in English at https://soundcloud.com/cmajpodcasts/190395-guide; entrevue en français au https://soundcloud.com/cmajpodcasts/190395-guide-fre

See related article at www.cmaj.ca/lookup/doi/10.1503/cmaj.191437

$\mathbf{T}$ his guideline from the Canadian Task Force on Preventive Health Care focuses on screening for thyroid dysfunction among asymptomatic nonpregnant adults in primary care beyond usual care and vigilance for signs and symptoms of thyroid dysfunction. Thyroid dysfunction is diagnosed based on abnormal levels of serum thyroid-stimulating hormone (TSH) and can be characterized as either hypo- or hyperthyroidism. Hypothyroidism results from impaired thyroid hormone production (i.e., thyroxine $\left[T_{4}\right]$ or triiodothyronine $\left[T_{3}\right]$ ), leading to elevated levels of TSH. Hypothyroidism is often caused by autoimmune disorders (e.g., Hashimoto thyroiditis) or occurs as a sequela of hyperthyroidism treatment, which can render the thyroid gland nonfunctional. ${ }^{1}$ Hyperthyroidism results from an overproduction of thyroid hormone, leading to the suppression of TSH. ${ }^{1}$ Causes of hyperthyroidism include Graves disease, toxic multinodular goitre and toxic adenoma. ${ }^{2}$

Signs and symptoms of thyroid dysfunction are variable between patients and often nonspecific. For hypothyroidism, symptoms may include tiredness, sensitivity to cold, dry skin, hair loss, weight gain and slowed movements and thoughts. ${ }^{1,3-6}$ For hyperthyroidism, symptoms may include sinus tachycardia, atrial fibrillation, hyperactivity or irritability, intolerance to heat, tremor and weight loss. ${ }^{1,2,7}$ Some people with thyroid dysfunction are asymptomatic. ${ }^{8}$

If left untreated, hypothyroidism may increase the risk of cardiac dysfunction, hypertension, dyslipidemia, cognitive impairment and, in rare cases, myxedema coma. ${ }^{3,9}$ Untreated hyperthyroidism may increase the risk of cardiac conditions (e.g., atrial fibrillation, heart failure) or bone fractures, and could lead to thyroid storm, an uncommon, life-threatening condition associated with tachycardia, extreme fatigue, fever and nausea. ${ }^{2,10}$

\section{KEY POINTS}

- The Canadian Task Force on Preventive Health Care strongly recommends against screening for thyroid dysfunction in asymptomatic nonpregnant adults.

- Screening for thyroid dysfunction in asymptomatic nonpregnant adults is not likely to confer clinical benefit, but could lead to unnecessary treatment for some patients and consume resources.

- Treating asymptomatic adults for screen-detected hypothyroidism may result in little to no difference in clinical outcomes.

- Clinicians should remain alert to signs and symptoms suggestive of thyroid dysfunction and investigate accordingly.

Minor variations in thyroid function as measured by abnormal levels of TSH are often self-limiting. Observational studies have reported that levels of TSH appear to revert to normal without treatment in $37 \%-62 \%$ of patients with initially elevated levels and $51 \%$ with initially low levels, particularly for milder cases of thyroid dysfunction (mean follow-up 32-60 mo). .11,12 $^{12}$

Screening is intended to detect thyroid dysfunction in asymptomatic patients in order to prevent adverse consequences of untreated thyroid dysfunction. ${ }^{13}$ Screening is done by performing a blood test for TSH. Abnormal levels of TSH are followed up with additional diagnostic testing that often includes blood tests to measure thyroid hormone levels or other tests (e.g., ultrasound) as warranted. An estimated $10 \%$ of Canadians aged 45 years or older report that they have been diagnosed with thyroid dysfunction, and prevalence is higher in women (16\%) than in men (4\%). ${ }^{14}$ Prevalence has also been reported to be higher in adults older than 85 years $(16 \%),{ }^{14}$ 
although evidence suggests that concentrations of TSH increase with age even in the absence of obvious thyroid dysfunction, and that age-specific reference intervals for TSH should be used to avoid unnecessary treatment. ${ }^{15}$ In addition to female sex and older age, risk factors for thyroid dysfunction include medications that might affect thyroid hormone levels (e.g., lithium, amiodarone); other autoimmune diseases (e.g., type 1 diabetes mellitus, Addison disease); previous surgery or radiation therapy on the thyroid gland, head or neck area; and a family history of thyroid disease..$^{1,5,9,16}$

Thyroid hormone replacement medication is used to treat hypothyroidism, ${ }^{1}$ whereas treatments for hyperthyroidism include antithyroid medication, radioiodine ablation or thyroid gland surgery. ${ }^{9}$

To make its recommendation, the task force considered evidence from systematic reviews on the benefits and harms of screening for asymptomatic thyroid dysfunction, treatment of asymptomatic thyroid dysfunction, and patient values and preferences.

\section{Scope}

This recommendation provides guidance to clinicians, policymakers and patients on screening for thyroid dysfunction among asymptomatic nonpregnant adults. These recommendations do not apply to patients with previously diagnosed thyroid disease or thyroid surgery; exposure to medications known to affect thyroid function; exposure to thyroid radioiodine therapy, or radiotherapy to the head or neck area; or pituitary or hypothalamic diseases.

\section{Methods}

The task force is an independent panel of clinicians and methodologists that makes recommendations on primary and secondary prevention in primary care (www.canadiantaskforce.ca). This recommendation was developed by a working group of 4 task force members (R.B., J.D., D.R. and B.T.) with scientific support from Public Health Agency of Canada staff.

The recommendation is based on systematic reviews ${ }^{17}$ on the effectiveness (i.e., benefits and harms) of screening for thyroid dysfunction in asymptomatic, nonpregnant adults; the effectiveness of treating screen-detected thyroid dysfunction in asymptomatic, nonpregnant adults; and patient values and preferences related to screening (see analytic framework, Appendix 1, available at www. cmaj.ca/lookup/suppl/doi:10.1503/cmaj.190395/-/DC2). The systematic reviews were based on a previous United States Preventive Services Task Force review ${ }^{18}$ with additional search terms included for patient values and preferences and all searches updated to July 25 , 2018. We excluded studies if they recruited patients with clinically obvious hypothyroidism or hyperthyroidism (e.g., Graves disease), or patients who were recently admitted to hospital.

The protocol (PROSPERO: CRD42016033622), systematic reviews ${ }^{17}$ and draft guideline were externally peer reviewed. Two clinical experts engaged with Public Health Agency of Canada staff to address technical and clinical considerations. Clinical experts are external to the task force and did not participate in guideline working group meetings or have input into, or vote on, task force recommendations.

The working group rated outcomes in accordance with the Grading of Recommendations, Assessment, Development and Evaluation (GRADE) approach. ${ }^{19} \mathrm{Clinical}$ outcomes that were rated critical for assessment in the systematic reviews were mortality (all-cause or cardiovascular), cardiovascular events (fatal and nonfatal, atrial fibrillation), fractures, thyroid-specific quality of life, cognitive function and harms from treatment. Additional clinical outcomes rated as important included physical, mental, or general well-being; fatigue or tiredness; and harms from screening (e.g., psychological effects, harms of workup, overdiagnosis and overtreatment). Intermediate outcomes (i.e., physiologic measures related to the screening intervention but not clinical outcomes in and of themselves ${ }^{20}$ ) rated as important were blood pressure, bone mineral density, cholesterol and weight change. ${ }^{17}$

We also used the GRADE approach to determine the certainty of the evidence and strength of the recommendation (Box 1). ${ }^{19}$ Appendix 2 (available at www.cmaj.ca/lookup/suppl/doi:10.1503/ cmaj.190395/-/DC2) provides the evidence-to-decision framework that the task force used to assess the balance between benefits and harms, patient values, resource use, feasibility, acceptability and equity in order to develop the recommendation. The entire task force reviewed and approved the recommendation.

The Knowledge Translation Program at St. Michael's Hospital (Toronto) developed the knowledge translation tool accompanying this guideline. The tool can be found on the task force website (www.canadiantaskforce.ca). The tool was informed by feedback from clinicians.

\section{Management of competing interests}

Funding for the task force is provided by the Public Health Agency of Canada. The task force does not consider the views of the funding body in developing its recommendations. All task force members and clinical experts are required to disclose financial and nonfinancial conflicts of interest, which are made available publicly on the task force website. All but 1 task force member declared no conflicts of interest for this guideline: R.G. declared a conflict related to the 2016 creation of non-industryfunded educational material on managing thyroid disease; he was not a member of the working group and chose not to vote on the guideline. Clinical experts are required to disclose any conflicts of interest at the outset of their participation, and annually thereafter. The 2 clinical experts (B.C. and A.Z.) involved with this guideline declared that they had no conflicts of interest for this guideline. Clinical experts did not participate in discussions on recommendations and did not vote on recommendations.

\section{Recommendation}

We recommend against screening asymptomatic nonpregnant adults aged 18 years and older for thyroid dysfunction in primary care settings (strong recommendation, low-certainty evidence).

A summary of the recommendation is available in Box 2 . 


\section{Box 1: Grading of recommendations}

Recommendations are graded according to the Grading of Recommendations Assessment, Development and Evaluation system (GRADE)..$^{19}$ Whether a recommendation is strong or conditional ${ }^{*}$ will depend on considerations such as certainty in estimated effects of an intervention, including magnitude, as well as estimates of how patients value and prioritize outcomes, variability of these estimates and wise use of resources.

\section{Strong recommendations}

Strong recommendations are those for which the Canadian Task Force for Preventive Health Care is confident that the desirable effects of an intervention outweigh its undesirable effects (strong recommendation for an intervention) or that the undesirable effects of an intervention outweigh its desirable effects (strong recommendation against an intervention). A strong recommendation implies that most people will be best served by the recommended course of action.

Strong recommendations are typically based on high-certainty evidence (i.e., high confidence in the estimate of the effect of an intervention). Strong recommendations may recommend in favour of an intervention (when there is high confidence of net benefit) or against an intervention (when there is high confidence of net harm). However, there are circumstances in which a strong recommendation may be considered based on low- or very low-certainty evidence or when there is absence of evidence or low-certainty evidence of benefit. ${ }^{21}$

When there is an absence of evidence to provide confidence that there is benefit from implementing a new prevention service or when a conclusion of possible benefit requires a high level of speculation on linkages of uncertain evidence, but there is high certainty that some patients would be harmed or scarce health care resources expended, the task force may make a strong recommendation against service implementation. This is consistent with the GRADE approach, in which strong recommendations are sometimes made with low-certainty evidence combined with high certainty of harm or resource implications, and with the value that the task force places on using scarce primary care resources wisely.

\section{Conditional recommendations}

Conditional recommendations are those for which the desirable effects probably outweigh the undesirable effects (conditional recommendation in favour of an intervention) or undesirable effects probably outweigh the desirable effects (conditional recommendation against an intervention) but appreciable uncertainty exists. Conditional recommendations are made when the certainty of evidence is lower, when the margin between desirable and undesirable consequences is small and the balance depends on patient values and preferences, or when there is high variability in the values and preferences of patients. Conditional recommendations may also be applied when the balance of cost and benefits is ambiguous, key stakeholders differ about the acceptability or feasibility of the implementation, or the effects on health equity are unclear.

In certain cases where a conditional recommendation for an intervention is made, clinicians are encouraged to engage in shared decision-making, to recognize that different choices will be appropriate for individual patients, and to help each person arrive at a management decision consistent with their values and preferences. Clinicians should recognize that different choices will be appropriate for different patients and that decisions must be consistent with each patient's values and preferences.

Knowledge translation tools are available on the task force website (www.canadiantaskforce.ca) to facilitate decisions that are evidence informed and aligned with an individual's priorities.

Evidence is graded as high-, moderate-, low- or very low-certainty, based on how likely further research is to change the task force's confidence in the estimate of effect.

*The task force previously used the term "weak recommendation," but has replaced this with the term "conditional recommendation," to improve understanding and facilitate implementation of guidance, based on feedback from clinician knowledge users. One reason for this change was the value that the task force places on shared decision-making, together with a need to clarify better when implementation of a recommendation depends on circumstances such as patient values, resource availability or other contextual considerations. Conditional recommendations based on patient values and preferences require clinicians to recognize that different choices will be appropriate for different patients and those decisions must be consistent with each patient's values and preferences.

\section{Screening for thyroid dysfunction}

The systematic reviews conducted to support the guideline did not find any eligible studies that directly assessed the benefits or harms of screening for thyroid dysfunction compared with not screening in asymptomatic nonpregnant adults. ${ }^{17}$

\section{Treatment of screen-detected thyroid dysfunction}

Because the task force found no studies comparing screening to no screening for thyroid dysfunction, we considered indirect evidence on the effectiveness of the treatment of asymptomatic thyroid dysfunction. ${ }^{17}$ The systematic review on treatment included studies that screened for thyroid dysfunction and followed only those participants who screened positive. In total, there were 22 eligible studies, including 19 randomized controlled trials (RCTs) and 3 cohort studies. In 14 RCTs (16 publications), nonpregnant adults who screened positive for hypothyroidism were randomized to receive either levothyroxine treatment or placebo, ${ }^{22-37}$ and 5 RCTs $^{38-42}$ and 3 cohort studies ${ }^{43-45}$ compared levothyroxine treatment to no treatment. The length of follow-up ranged from 3 to 36 months for the RCTs and between 5.0 and 7.6 years (median)
Box 2: Summary of recommendation for clinicians, policy-makers and patients

We recommend against screening * asymptomatic nonpregnant adults aged 18 years and older for thyroid dysfunction in primary care settings (strong recommendation, low-certainty evidence).

This recommendation does not apply to adults who have the following risk factors for thyroid dysfunction:

- Individuals who have had a previously diagnosed thyroid disease or surgery;

- Individuals receiving thyroid medications or medications that may affect thyroid function (i.e., lithium, amiodarone);

- Individuals with previous or ongoing exposure to thyroid radioiodine therapy or head and neck radiotherapy; or

- Individuals with pituitary or hypothalamic diseases.

*Screening refers to measuring levels of thyroid-stimulating hormone (TSH) in patients with no apparent signs or symptoms of thyroid dysfunction. Screening differs from measuring levels of TSH among patients who are experiencing signs and symptoms (e.g., fatigue, weight gain, menstrual irregularities, goitre) or are at risk for thyroid dysfunction due to secondary causes (e.g., pituitary or hypothalamic diseases) to investigate possible thyroid dysfunction. 
for the cohort studies. Patients were recruited via populationbased screening in 3 of the RCTs, ${ }^{24,25,32,42}$ and in the remainder of the trials and cohort studies, participants were from primary care as well as specialized and nonspecified outpatient clinics. Studies took place in Europe, the Middle East, South America and Asia. We did not identify any studies reporting on treatment for screendetected hyperthyroidism. We synthesized data narratively because of the clinical and methodological variability among the included studies, similar to the approach used in the United States Preventive Services Task Force review. ${ }^{18}$

\section{Clinical outcomes}

Mortality (all-cause and cardiovascular) and cardiovascular events One RCT $(n=737)^{34}$ assessed the effect of treatment in participants aged 65 years and older on mortality (all-cause or cardiovascular; low- and very low-certainty evidence, respectively), and cardiovascular events (fatal and nonfatal, atrial fibrillation; low-certainty evidence) and reported no statistically significant differences between treatment and placebo (Appendix 3, Supplemental Table 1, available at www.cmaj.ca/lookup/suppl/doi:10.1503/cmaj.190395/-/ DC2). Very low-certainty evidence on adults aged $40-70$ years and younger than 65 years from 2 cohort studies was used to inform outcomes for younger adults. ${ }^{43,45}$ These 2 studies $^{43,45}$ reported a minimal reduction in all-cause mortality and equivocal results for cardiovascular outcomes (Appendix 3, Supplemental Table 1). Two cohort studies ${ }^{43,44}$ reported no statistically significant differences in all-cause mortality or cardiovascular events for women or men between those treated and not treated with levothyroxine for screen-detected hypothyroidism.

\section{Cognitive function}

Low-certainty evidence from 3 RCTs $(n=759)^{25,32,34}$ reported on 22 cognitive function outcome analyses, stemming from 20 unique tests (i.e., 2 tests were included in 2 separate RCTs). Only 2 tests (the Composite Cognitive Score and the Speed and Capacity of Language Processing Test) showed statistically significant treatment effects over placebo (Appendix 3, Supplemental Table 1).

\section{Fractures and quality of life measures}

The task force found no statistically significant differences between treatment and no treatment or placebo for any of the remaining clinical outcomes: fractures (low-certainty evidence from 1 RCT), ${ }^{34}$ thyroid-specific quality of life (moderate-certainty evidence from $1 \mathrm{RCT}),{ }^{34}$ mental well-being (moderate-certainty evidence from 4 RCTs), ${ }^{25,31-33}$ physical well-being (moderate-certainty evidence from $1 \mathrm{RCT}$ ), ${ }^{34}$ general well-being (moderate-certainty evidence from 3 RCTs), ${ }^{25,33,34}$ or fatigue or tiredness (moderate-certainty evidence from 1 RCT) ${ }^{34}$ (Appendix 3, Supplemental Table 1).

\section{Intermediate outcomes}

\section{Blood pressure}

There were no statistically significant differences for blood pressure in 8 studies that included that outcome (moderatecertainty evidence). ${ }^{26,28-30,34,37,41,42}$

\section{Bone mineral density}

We found no studies that addressed bone mineral density.

\section{Weight change}

Ten RCTs $22-24,26,28-30,34,37,39$ found no differences in weight change, $1 \mathrm{RCT}^{42}$ did not report the difference between groups, and $1 \mathrm{RCT}^{36}$ reported a mean increase in body mass index measurement among the treatment group (moderate-certainty evidence).

\section{Cholesterol}

Cholesterol outcomes were reported separately as total cholesterol, high-density lipoprotein, low-density lipoprotein and tri-

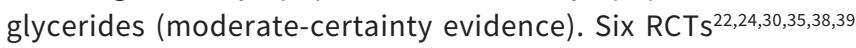
reported no differences in total cholesterol levels, and $4 \mathrm{RCTs}^{26,27,29,42}$ reported a decrease in total cholesterol levels among those treated compared with the control group. Nine $\mathrm{RCTs}^{22,24,26,27,29,30,35,38,39}$ reported no differences in high-density lipoprotein and $1 \mathrm{RCT}^{42}$ did not report the difference between groups. Eight RCTs $\mathrm{s}^{22,24,26,27,30,35,38,39}$ reported no differences in lowdensity lipoprotein levels, $1 \mathrm{RCT}^{42}$ did not report the difference between groups, and $1 \mathrm{RCT}^{29}$ reported that treatment for subclinical hypothyroidism led to a mean decrease in low-density lipoprotein levels. Nine RCTs $22,24,26,27,29,30,35,38,42$ reported no differences in triglyceride levels and $1 \mathrm{RCT}^{39}$ reported a decrease in mean triglyceride levels in the treatment group.

Although there were few statistically significant reductions in lipid markers, the point estimate mean differences in levels of total cholesterol, low-density lipoprotein and triglycerides suggest some improvement with treatment in 23 of 27 analyses $^{16}$ (point estimate ranges for total cholesterol: -1.07 to $0.00 \mathrm{mmol} / \mathrm{L}$; low-density lipoprotein: -1.23 to $0.11 \mathrm{mmol} / \mathrm{L}$; triglycerides: -1.94 to $0.12 \mathrm{mmol} / \mathrm{L}$ ).

\section{Harms of treatment}

Low-certainty evidence from 7 RCTs $26,30,32,34,35,40,42$ reported no statistically significant differences in adverse outcomes (e.g., adverse events, symptoms, adverse effects) of treatment between groups, treated and not treated, for screen-detected hypothyroidism. Furthermore, the proportion of withdrawals owing to adverse outcomes was similar between the control $(0 \%-14.3 \%)$ and intervention groups (0\%-9.6\%). ${ }^{26,32,35,42}$

\section{Patient values and preferences}

The systematic reviews identified no studies that reported on patients' values and preferences related to screening for thyroid dysfunction. ${ }^{17}$

\section{Resource use}

The total resource requirements of screening asymptomatic adults for thyroid dysfunction were not evaluated via systematic review, but would include the unit costs of laboratory tests for TSH (about \$5-14 each) ${ }^{46-48}$ and additional financial costs borne by patients and the provinces and territories related to diagnostic tests, provider fees, treatment, monitoring and lifelong medical follow-up for cases of identified thyroid dysfunction. The task force did not assess the cost-effectiveness of screening for thyroid dysfunction. In the judgment of the task force, given the 
lack of evidence of clinical effectiveness, financial costs of screening asymptomatic adults would represent an undesirable consequence for the health care system.

\section{Feasibility, acceptability and equity}

No formal programs exist in Canada to screen for thyroid dysfunction among adults, although many clinicians routinely order TSH tests for asymptomatic patients. One study from Toronto found that $71 \%$ of 135243 patients (aged 20 yr or older) without known thyroid disease and not on thyroid medication had their levels of TSH tested at least once over a 2-year period, and $92 \%$ of these tests came back normal. ${ }^{49} \mathrm{~A}$ recommendation against screening for thyroid dysfunction may represent a change in current practice for some clinicians; in the judgment of the task force, this would reduce unnecessary tests and burden on patients. Given the lack of evidence of benefit, in the judgment of the task force, this recommendation is feasible and acceptable to most clinicians, policy-makers and patients and would neither increase nor decrease equity.

\section{Rationale}

The task force found no studies that directly evaluated the benefits and harms of screening for thyroid dysfunction among asymptomatic nonpregnant adults. We identified no studies that reported on treatment for screen-detected hyperthyroidism. On balance, evidence on the treatment of screen-detected hypothyroidism did not indicate improvement for the clinical outcomes examined. The task force placed greater value on low-certainty evidence from 1 RCT indicating no effect on all-cause mortality than on very lowcertainty evidence from 2 cohort studies, which showed a very small reduction in mortality among people aged $40-70$ years and younger than 65 years, and 1 cohort study that showed no benefit.

The task force believes that asymptomatic adults would be unlikely to choose to be screened for thyroid dysfunction if they were aware of the lack of evidence of clinical effectiveness beyond usual care and vigilance; the potential for overdiagnosis, meaning the diagnosis of transient thyroid dysfunction or dysfunction that would never manifest in symptoms; the need for follow-up testing and longterm monitoring; and increased treatment burden..$^{50}$ Therefore, the task force recommends against screening asymptomatic nonpregnant adults for thyroid dysfunction given the lack of demonstrated benefit on critical and important clinical outcomes, along with the financial costs to patients and the health care system.

\section{Implementation}

This recommendation applies only to screening asymptomatic nonpregnant adults for thyroid dysfunction. Clinicians should be aware of symptoms, signs and conditions associated with thyroid dysfunction so patients with these can be tested, particularly symptomatic postmenopausal women (given the higher prevalence of hypothyroidism in that population), ${ }^{9}$ and those with atrial fibrillation, when hyperthyroidism may be suspected. We did not find evidence on the effectiveness of screening for thyroid dysfunction specifically among patients with other chronic conditions, but there may be different reasons to test their levels of TSH to optimize management of their primary condition. These recommendations do not apply to patients with previously diagnosed thyroid disease or thyroid surgery; exposure to medications known to affect thyroid function (e.g., lithium, amiodarone); exposure to thyroid radioiodine therapy, or radiotherapy to the head or neck area; or pituitary or hypothalamic diseases.

\section{Monitoring and evaluation}

Indicators of the uptake of this recommendation against screening asymptomatic nonpregnant adults for thyroid dysfunction could be clinician awareness of this recommendation and reduction in regular tests for TSH among asymptomatic patients presenting for periodic health assessments. The task force will monitor evidence related to this guideline and will update the recommendation if new evidence becomes available that could influence its direction or strength.

\section{Other guidelines}

Variation currently exists among guidelines from other organizations (Table 1). The British Columbia Ministry of Health ${ }^{51}$ and Toward Optimized Practice ${ }^{52}$ from Alberta recommend against testing for TSH in asymptomatic patients. The United States Preventive Services Task Force did not provide a recommendation either for or against screening for thyroid dysfunction because of its determination that there was insufficient evidence to assess the balance of benefits and harms. ${ }^{5} \mathrm{~A}$ joint recommendation by the American Thyroid Association and American Association of Clinical Endocrinologists suggests that screening for hypothyroidism should be considered in patients older than 60 years. ${ }^{53}$

Table 1: National and international recommendations on screening for thyroid dysfunction

\section{Guideline group}

British Columbia Ministry of Health ${ }^{51}$

Toward Optimized Practice ${ }^{52}$

United States Preventive Services Task Force ${ }^{5}$

American Thyroid Association and American Association of Clinical Endocrinologists ${ }^{53}$

\section{Recommendation}

Routine thyroid function testing is not recommended in asymptomatic adults. However, testing may be indicated when nonspecific signs and symptoms are present in patients at risk for thyroid disease.

Do not test patients who are asymptomatic, seemingly healthy, having a periodic exam.

The United States Preventive Services Task Force concludes that the current evidence is insufficient to assess the balance of benefits and harms of screening for thyroid dysfunction in nonpregnant, asymptomatic adults.

Screening for hypothyroidism should be considered in patients older than age 60 years. This recommendation was downgraded because there is strong evidence that hypothyroidism is common in this group but insufficient evidence of benefit or cost-effectiveness. 


\section{Gaps in knowledge}

Despite the frequency of testing for TSH in Canada, there are no trials that directly assess the benefits and harms of screening for thyroid dysfunction versus not screening in asymptomatic nonpregnant adults. In addition, the task force found no evidence on the effectiveness of specifically screening patients with other concomitant conditions (e.g., cardiovascular diseases, type 1 diabetes mellitus or other autoimmune diseases).

\section{Conclusion}

The task force found no trial evidence on screening versus not screening asymptomatic nonpregnant adults for thyroid dysfunction. Low-certainty evidence suggests that treatment of screen-detected hypothyroidism among nonpregnant adults is unlikely to provide meaningful improvement on clinical outcomes reviewed by the task force. Screening will also result in overuse of resources without a demonstrated benefit. Therefore, the task force strongly recommends against screening for thyroid dysfunction in asymptomatic nonpregnant adults.

\section{References}

1. Jameson J, Mandel S, Weetman A. Disorders of the thyroid gland. In: Kasper D, Fauci A, Hauser S, editors. Harrison's principles of internal medicine. 19th ed. New York: McGraw-Hill; 2014.

2. Kravets I. Hyperthyroidism: diagnosis and treatment. Am Fam Physician 2016;93:363-70.

3. Gaitonde DY, Rowley K, Sweeney L. Hypothyroidism: an update. Am Fam Physician 2012;86:244-51.

4. Gillett M. Subclinical hypothyroidism: subclinical thyroid disease: scientific review and guidelines for diagnosis and management: JAMA 2004;291:228-38. Clin Biochem Rev 2004;25:191-4.

5. LeFevre ML, Siu AL, Bibbins-Domingo K, et al. Screening for thyroid dysfunction: U.S. Preventive Services Task Force recommendation statement. Ann Intern Med 2015;162:641-50.

6. Chaker L, Bianco A, Jonklaas J, et al. Hypothyroidism. Lancet 2017;390:1550-62.

7. Trivalle $C$, Doucet J, Chassagne $P$, et al. Differences in the signs and symptoms of hyperthyroidism in older and younger patients. J Am Geriatr Soc 1996;44:50-3.

8. Canaris GJ, Manowitz N, Mayor G, et al. The Colorado Thyroid Disease Prevalence Study. Arch Intern Med 2000;160:526-34.

9. Surks MI, Ortiz E, Daniels GH, et al. Subclinical thyroid disease: scientific review and guidelines for diagnosis and management. JAMA 2004;291:228-38.

10. Vestergaard P, Mosekilde L. Fractures in patients with hyperthyroidism and hypothyroidism: a nationwide follow-up study in 16249 patients. Thyroid 2002;12:411-9.

11. Díez J, Iglesias P. Spontaneous subclinical hypothyroidism in patients older than 55 years: An analysis of natural course and risk factors for the development of overt thyroid failure. J Clin Endocrinol Metab 2004;89:4890-7.

12. Meyerovitch J, Rotman-Pikielny $P$, Sherf M, et al. Serum thyrotropin measurements in the community: five-year follow-up in a large network of primary care physicians. Arch Intern Med 2007;167:1533-8.

13. Benefits and risks of screening tests. Bethesda (MD): U.S. National Library of Medicine; 2016. Available: www.ncbi.nlm.nih.gov/pubmedhealth/PMH0072602/ (accessed 2017 Dec. 12).

14. Statistics Canada. Table 105-1200 - Healthy aging indicators, by age group and sex, household population aged 45 and over, Canada and provinces, occasional, CANSIM (database). Ottawa: Statistics Canada; 2010. Available: http://www5. statcan.gc.ca/cansim/a26?lang=eng\&id=1051200 (accessed 2017 Dec. 12).

15. Vadiveloo T, Donnan PT, Murphy MJ, et al. Age- and gender-specific TSH reference intervals in people with no obvious thyroid disease in Tayside, Scotland: the Thyroid Epidemiology, Audit, and Research Study (TEARS). J Clin Endocrinol Metab 2013;98:1147-53.

16. Vanderpump MPJ. The epidemiology of thyroid disease. Br Med Bull 2011;99:39-51.

17. Reyes Domingo F, Avey MT, Doull M. Screening for thyroid dysfunction and treatment of screen-detected thyroid dysfunction in asymptomatic, communitydwelling adults: a systematic review. Syst Rev. In press.
18. Rugge JB, Bougatsos C, Chou R. Screening and treatment of thyroid dysfunction: an evidence review for the U.S. Preventive Services Task Force. Ann Intern Med 2015;162:35-45.

19. Schünemann H, Brożek J, Guyatt G, et al., editors. GRADE Handbook: Handbook for grading the quality of evidence and the strength of recommendations using the GRADE approach. Updated October 2013. GRADE Working Group; 2013. Available: http://gdt.guidelinedevelopment.org/app/handbook/handbook. html (accessed 2016 Nov. 9).

20. Wolff TA, Krist AH, LeFevre M, et al. Update on the methods of the U.S. Preventive Services Task Force: linking intermediate outcomes and health outcomes in prevention. Am J Prev Med 2018;54(1S1):S4-S10.

21. Andrews JC, Schünemann HJ, Oxman AD, et al. GRADE guidelines: 15. going from evidence to recommendation-determinants of a recommendation's direction and strength. J Clin Epidemiol 2013;66:726-35.

22. Caraccio N, Ferrannini E, Monzani F. Lipoprotein profile in subclinical hypothyroidism: response to levothyroxine replacement, a randomized placebocontrolled study. J Clin Endocrinol Metab 2002;87:1533-8.

23. Caraccio N, Natali A, Sironi A, et al. Muscle metabolism and exercise tolerance in subclinical hypothyroidism: a controlled trial of levothyroxine. J Clin Endocrinol Metab 2005;90:4057-62.

24. Iqbal A, Jorde R, Figenschau Y. Serum lipid levels in relation to serum thyroid-stimulating hormone and the effect of thyroxine treatment on serum lipid levels in subjects with subclinical hypothyroidism: the Tromso Study. $J$ Intern Med 2006;260:53-61.

25. Jorde R, Waterloo K, Storhaug $\mathrm{H}$, et al. Neuropsychological function and symptoms in subjects with subclinical hypothyroidism and the effect of thyroxine treatment. J Clin Endocrinol Metab 2006;91:145-53.

26. Liu P, Liu R, Chen X, et al. Can levothyroxine treatment reduce urinary albumin excretion rate in patients with early type 2 diabetic nephropathy and subclinical hypothyroidism? A randomized double-blind and placebo-controlled study. Curr Med Res Opin 2015;31:2233-40.

27. Mikhail GS, Alshammari SM, Alenezi MY, et al. Increased atherogenic low-density lipoprotein cholesterol in untreated subclinical hypothyroidism. Endocr Pract 2008;14:570-5.

28. Monzani F, Di Bello V, Caraccio N, et al. Effect of levothyroxine on cardiac function and structure in subclinical hypothyroidism: a double blind, placebocontrolled study. J Clin Endocrinol Metab 2001;86:1110-5.

29. Monzani F, Caraccio N, Kozakowa M, et al. Effect of levothyroxine replacement on lipid profile and intima-media thickness in subclinical hypothyroidism: a double-blind, placebo-controlled study. J Clin Endocrinol Metab 2004; 89:2099-106.

30. Nagasaki T, Inaba M, Yamada S, et al. Decrease of brachial-ankle pulse wave velocity in female subclinical hypothyroid patients during normalization of thyroid function: a double-blind, placebo-controlled study. Eur J Endocrinol 2009;160:409-15.

31. Najafi L, Malek M, Hadian A, et al. Depressive symptoms in patients with subclinical hypothyroidism-the effect of treatment with levothyroxine: a doubleblind randomized clinical trial. Endocr Res 2015;40:121-6.

32. Parle J, Roberts L, Wilson S, et al. A randomized controlled trial of the effect of thyroxine replacement on cognitive function in community-living elderly subjects with subclinical hypothyroidism: the Birmingham Elderly Thyroid study. $J$ Clin Endocrinol Metab 2010;95:3623-32.

33. Reuters VS, Almeida CP, Teixeira PFS, et al. Effects of subclinical hypothyroid ism treatment on psychiatric symptoms, muscular complaints, and quality of life. Arq Bras Endocrinol Metabol 2012;56:128-36.

34. Stott DJ, Rodondi N, Kearney PM, et al. Thyroid hormone therapy for older adults with subclinical hypothyroidism. N Engl J Med 2017;377:e20.

35. Teixeira PF, Reuters VS, Ferreira MM, et al. Lipid profile in different degrees of hypothyroidism and effects of levothyroxine replacement in mild thyroid failure. Transl Res 2008;151:224-31.

36. Teixeira PF, Reuters VS, Ferreira MM, et al. Treatment of subclinical hypothyroidism reduces atherogenic lipid levels in a placebo-controlled doubleblind clinical trial. Horm Metab Res 2008;40:50-5.

37. Yazici M, Gorgulu S, Sertbas Y, et al. Effects of thyroxin therapy on cardiac function in patients with subclinical hypothyroidism: index of myocardial performance in the evaluation of left ventricular function. Int J Cardiol 2004;95:135-43.

38. Cabral MD, Teixeira P, Soares D, et al. Effects of thyroxine replacement on endothelial function and carotid artery intima-media thickness in female patients with mild subclinical hypothyroidism. Clinics (Sao Paulo) 2011;66:1321-8.

39. Duman D, Sahin S, Esertas K, et al. Simvastatin improves endothelial function in patents with subclinical hypothyroidism. Heart Vessels 2007;22:88-93.

40. Fadeyev VV, Sytch J, Kalashnikov V, et al. Levothyroxine replacement therapy in patients with subclinical hypothyroidism and coronary artery disease. Endocr Pract 2006;12:5-17. 
41. Mainenti MRM, Vigário PS, Teixeira PFS, et al. Effect of levothyroxine replacement on exercise performance in subclinical hypothyroidism. J Endocrinol Invest 2009;32:470-3.

42. Zhao M, Liu L, Wang F, et al. A worthy finding: decrease in total cholesterol and low-density lipoprotein cholesterol in treated mild subclinical hypothyroidism. Thyroid 2016;26:1019-29.

43. Andersen MN, Olsen A-S, Madsen JC, et al. Levothyroxine substitution in patients with subclinical hypothyroidism and the risk of myocardial infarction and mortality. PLoS One 2015;10:e0129793.

44. Andersen MN, Olsen A-S, Madsen JC, et al. Long-term outcome in levothyroxine treated patients with subclinical hypothyroidism and concomitant heart disease. J Clin Endocrinol Metab 2016;101:4170-7.

45. Razvi S, Weaver JU, Butler TJ, et al. Levothyroxine treatment of subclinical hypothyroidism, fatal and nonfatal cardiovascular events, and mortality. Arch Intern Med 2012;172:811-7.

46. Thyroid stimulating hormone. Calgary: Calgary Laboratory Services; 2018. Available: www.calgarylabservices.com/lab-services-guide/lab-tests/ AlphabeticalListing/T/Thyroid-Stimulating-Hormone.htm (accessed 2018 Feb. 13).

47. Schedule of fees: for the laboratory services outpatient: payment schedule. Vancouver: British Columbia Ministry of Health; revised 2017 July 31. Available: www2.gov.bc.ca/assets/gov/health/practitioner-pro/laboratory-services/ laboratory_services_-_schedule_of_fees.pdf (accessed 2018 Mar. 21).
48. Birk-Urovitz E, Del Giudice E, Meaney C, et al. Use of thyroid-stimulating hormone tests for identifying primary hypothyroidism in family medicine patients. Can Fam Physician 2017;63:e389-94.

49. Greiver M, Mclsaac W, DelGiudice L, et al. Patterns of thyroid screening and case detection in patients without thyroid disease in Canadian primary care [poster]. North American Primary Care Research Group - 2017 Practice-Based Research Network (PBRN) Conference; 2017 June 22-23; Bethesda (MD).

50. Eton DT, Ramalho de Oliveira D, Egginton J, et al. Building a measurement framework of burden of treatment in complex patients with chronic conditions: a qualitative study. Patient Relat Outcome Meas 2012;3:39-49.

51. Thyroid function testing in the diagnosis and monitoring of thyroid function disorder. Vancouver: British Columbia Ministry of Health Services, Guidelines and Protocols Advisory Committee; 2018. Available: https://www2. gov.bc.ca/gov/content/health/practitioner-professional-resources/bc -guidelines/thyroid-testing (accessed 2019 Sept. 18).

52. Toward Optimized Practice Endocrine Working Group. Investigation and man agement of primary thyroid dysfunction clinical practice guideline. Edmonton: Toward Optimized Practice; 2014; Available: www.topalbertadoctors.org/ download/350/thyroid_guideline.pdf (accessed 2017 Dec. 8).

53. Garber JR, Cobin R, Gharib H, et al. Clinical practice guidelines for hypothyroid ism in adults: cosponsored by the American Association of Clinical Endocrinologists and the American Thyroid Association. Endocr Pract 2012;18:988-1028.
Authors: Richard Birtwhistle MD MSc, Kate Morissette MSc, James A. Dickinson MBBS, Donna L. Reynolds MD MSc, Marc T. Avey PhD, Francesca Reyes Domingo MHSc, Rachel Rodin MD, Brett D. Thombs PhD; Canadian Task Force on Preventive Health Care

Competing interests: No members of the working group declared competing interests. One member of the task force, Roland Grad, declared a conflict of interest related to the 2016 development of an educational video on thyroid dysfunction, not funded by industry. He was not a member of the working group and chose not to vote on the guideline. All other task force members declared that they had no conflicts of interest.

This article has been peer reviewed.

Affiliations: Departments of Family Medicine and Public Health Sciences (Birtwhistle), Queen's University, Kingston, Ont.; Public Health Agency of Canada (Morissette, Avey, Reyes Domingo, Rodin), Ottawa, Ont.; Departments of Family Medicine and Community Health Sciences (Dickinson), University of Calgary, Alta.; Dalla Lana School of Public Health and Department of Family and Community Medicine (Reynolds), University of Toronto, Ont.; Department of Psychiatry (Thombs), Jewish General Hospital and McGill University, Montréal, Que.

Guideline Writing Group: Richard Birtwhistle (voting task force member), Kate Morissette (nonvoting science team member), James A. Dickinson (voting task force member), Donna L. Reynolds (voting task force member), Marc T. Avey (nonvoting science team member), Francesca Reyes Domingo (nonvoting science team member), Rachel Rodin (nonvoting science team member), Brett D. Thombs (voting task force member).

Collaborating Members of the Canadian Task Force on Preventive Health Care: Heather Colquhoun, Roland Grad, Stéphane Groulx, Scott Klarenbach, Michael Kidd, Christina Korownyk, Eddy Lang, John C. LeBlanc, Ainsley Moore, Navindra Persaud, John J. Riva, Guylène Thériault and Brenda J. Wilson. The complete list of all current members of the Canadian Task Force on Preventive Health Care is available at www.canadiantaskforce.ca/about/members.

Contributors: Richard Birtwhistle, Kate Morissette, James A. Dickinson, Donna L. Reynolds, Marc T. Avey, Francesca Reyes Domingo, Rachel Rodin, and Brett D. Thombs contributed substantially to the study concept and design, interpretation of the evidence and critical revision of the guideline draft. Kate Morissette and Marc T. Avey provided scientific and logistical support to the writing group. Richard Birtwhistle,
Heather Colquhoun, James A. Dickinson, Stéphane Groulx, Scott Klarenbach, Eddy Lang, John C. LeBlanc, Ainsley Moore, Donna L. Reynolds, Guylène Thériault, Brett $D$. Thombs and Brenda J. Wilson drafted the recommendation. Richard Birtwhistle, Kate Morissette, Marc T. Avey, Francesca Reyes Domingo and Rachel Rodin drafted the guideline statement. All of the named authors gave final approval of the version of the guideline to be published and agreed to be accountable for all aspects of the work. All collaborating members of the Canadian Task Force on Preventive Health Care (Heather Colquhoun, Roland Grad, Stéphane Groulx, Scott Klarenbach, Michael Kidd, Christina Korownyk, Eddy Lang, John C. LeBlanc, Ainsley Moore, Navindra Persaud, John J. Riva, Guylène Thériault and Brenda J. Wilson) also made contributions to the development of the guideline and all gave final approval of the version of the guideline to be published.

Funding: Funding for the Canadian Task Force on Preventive Health Care is provided by the Public Health Agency of Canada. The task force does not consider the views of the funding body in developing its recommendations. The views expressed in this article are those of the task force and do not necessarily represent those of the Public Health Agency of Canada.

Acknowledgements: The authors thank the team at the Global Health and Guidelines Division at the Public Health Agency of Canada (Marion Doull and Eva Graham) for its support on this guideline and the Knowledge Translation Program, Li Ka Shing Knowledge Institute, St. Michael's Hospital for its contributions to knowledge translation work related to this guideline. The authors also thank Bernard Corenblum, University of Calgary, Calgary, Alta., and Afshan Zahedi, University of Toronto, Toronto, Ont., for assisting as clinical experts during the development of this guideline. Clinical experts who have assisted the task force in the guideline process may or may not agree with the task force recommendations. Additionally, the authors thank peer-reviewers and organizational stakeholders who provided feedback on the draft guideline, including Mike Allan, College of Family Physicians of Canada; Patrick Chu, Canadian Geriatrics Society; Heather Lochnan, Canadian Society of Endocrinology and Metabolism; Hortensia Mircescu, Canadian Society of Endocrinology and Metabolism; Deric Morrison, Thyroid Foundation of Canada; Ferhan Siddiqi, Canadian Society of Endocrinology and Metabolism; Jay Silverberg, University of Toronto; Ian Shrier, McGill University; Howard Tracer, Agency for Healthcare Research and Quality; and Jody Woolfrey, Canadian Geriatrics Society.

Correspondence to: Canadian Task Force on Preventive Health Care, info@canadiantaskforce.ca 